\title{
Performance of Commercial MIMO Access Point in Distributed Antenna System with Different Fiber Lengths
}

\author{
Yuting Fan ${ }^{1,2}$, Anthony E.Aighobahi ${ }^{2}$, Nathan J.Gomes ${ }^{2 *}$, Kun Xu ${ }^{1 *}$ \\ ${ }^{1}$ State Key Laboratory of Information Photonics and Optical Communications, Beijing University of Posts and \\ Telecommunications, Beijing, 100876, P. R. China \\ Email: xukun@bupt.edu.cn \\ ${ }^{2}$ Broadband and Wireless Communications Group, School of Engineering and Digital Arts, University of Kent, Kent, UK \\ Email: N.J.Gomes@kent.ac.uk
}

\begin{abstract}
In this paper, we experimentally investigate the throughput of IEEE 802.11n $2 \times 2$ multiple-input-multiple-output (MIMO) signals in a radio-over-fiber-based distributed antenna system (DAS) with different fiber links. Both a MIMO-supported access point (AP) and a spatial-diversity-supported AP were separately employed in the experiments. Throughput measurements were carried out with wireless users at different locations in a typical office environment. The results indicate that MIMO signals can maintain high throughput when the fiber length difference between the two remote antenna units (RAUs) is under $100 \mathrm{~m}$ and falls quickly when the length difference is greater. For the spatial diversity signals, high throughput can be maintained even when the difference is $150 \mathrm{~m}$.
\end{abstract}

Keywords-Distributed antenna system; IEEE 802.11n; MIMO;

\section{INTRODUCTION}

Distributed antenna systems (DASs) using radio-over-fiber (RoF) links have been demonstrated as a commonly used infrastructure solution in in-building environments [1-2]. In a RoF-based DAS [3], multiple spatially separated remote antenna units (RAUs) are fiber-connected to a central unit (CU) where base station or access point facilities are placed. RoFbased DAS can be used to distribute WLAN signals and mobile telephony signals (3G, Wimax, Long Term Evolution (LTE), etc.). It can also combine the multiple radio streams together for neutral host providers due to its transparency, broadband, low attenuation and cost efficient features. In DAS applications, the base station facility in the $\mathrm{CU}$ is usually connected to multiple RAUs to extend the indoor wireless coverage of the base station and to share the hardware and bandwidth resource. By increasing the number of RAUs, the radio signal propagation distance is much shorter and radio signals are supposed to experience better channel quality since there is lower path loss, penetration loss and shadowing, which can significantly improve the system capacity and coverage. Furthermore, upgrading and re-configuration can be more easily performed in this centralized architecture. These features make RoF-based DAS attractive to achieve a costeffective, scalable and flexible network.
Meanwhile, multiple-input-multiple-output (MIMO) technology has gained much attentions in wireless communication systems because of its high-speed, highquality and high-reliability advantages. The integration of MIMO and RoF, which can improve the throughput performance and extend the wireless coverage by increasing the received RF power and the antenna separation, has also been reported in previous research [4-5]. It has also been shown in $[7,8]$ that a major issue with DAS when distributing WLAN signals over a fiber system is the effect of different fiber lengths on the received OFDM signal. This case is in a simulcast DAS, when a single AP (BS) feeds multiple RAUs. A similar situation should occur in MIMO-RoF systems, where multiple transmitters and receivers are employed in the system. The CU or the clients need to align the OFDM signals and combine the different spatial streams before demodulation. For a re-configurable in-building network design in practice, it becomes necessary to ensure equivalent fiber length for every link, especially considering the reuse of the large installed base of fiber links.

OFDM in both IEEE $802.11 \mathrm{~g}$ and $802.11 \mathrm{n}$ has a symbol duration of 4 usec. The guard interval is $0.8 \mathrm{usec}$ for $802.11 \mathrm{~g}$ and 0.4 usec for $802.11 \mathrm{n}$ in default mode. In an ideal wireless environment, the guard interval allows the path difference up to 400 feet $(122 \mathrm{~m})$ for $802.11 \mathrm{n}[9]$, but normally it cannot reach such a large difference. In a mixed optical-wireless channel, as the speed of light in vacuum is higher than in the fiber, the ideal maximum distance will be less than 400 feet.

In this paper, we experimentally investigate the throughput of an IEEE 802.11n 2x2 MIMO-over-fiber system with different fiber links. We compare the results with the performance of a spatial-diversity-supported AP, which has been reported to provide a better performance against fiber length difference effects in [6].

The experimental setup for the proposed system is described in Section II, whilst the results analysis is shown in Section III. Conclusions follow in Section IV. 


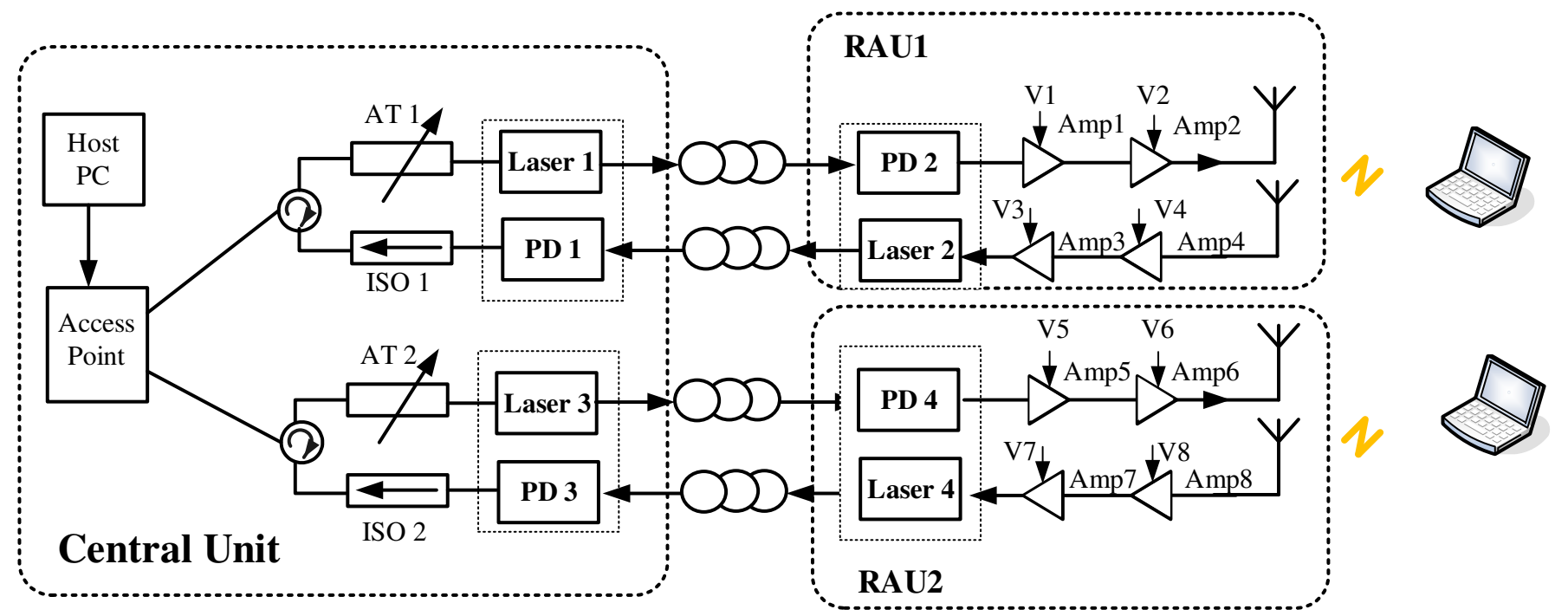

Fig. 1. The experiment set-up. $\mathrm{PD}=$ Photodetector, $\mathrm{AT}=$ Attenuator, V=Voltage, $\mathrm{ISO}=$ Isolator, Amp=Amplifier.

\section{EXPERIMENTAL SETUP}

Fig.1 and Fig.2 show the Dual-RAU experimental setup and the experiment layout. The CU and RAUs were placed in separated rooms to make sure that the signal from the AP cannot wirelessly arrive at the area covered by the RAUs. The AP connects to a host PC using a 100Mbps Ethernet link. Two antenna ports of the AP and each RAU are connected by RoF links. In the downlink, the RF signals transmitted from the AP are converted into optical signals by the distributed feedback (DFB) laser diode (LD) and the resulting optical signals are transported over the SMF links. At the RAUs, the optical signals are detected by the photodiodes and amplified for transmission over the wireless path. Reverse processes occur in the uplink. The power at the input of the lasers was set at $2.5 \mathrm{dBm}$ achieved by attenuators, at which point, the Error Vector Magnitude (EVM) of the whole downlink can reach the best performance. Amplifiers were biased at different voltages, so that they did not operate in their non-linear regions. The output power before the antennas was set at $1 \mathrm{dBm}$. In all experiments described in the next section, the output power was set at this level, removing the effect of low received power.

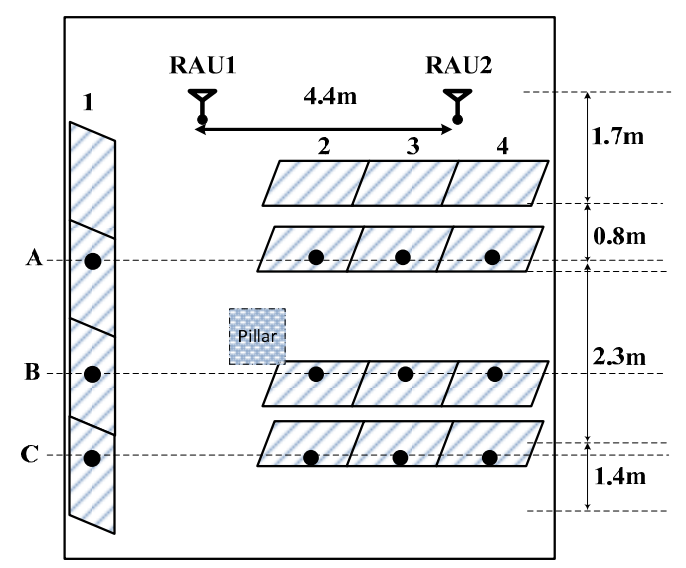

Fig. 2. Experiment layout
In the testing room, the distance between the two RAUs was set to $4.4 \mathrm{~m}$.. The larger distance can reduce the spatial correlation and provide higher received RF power for the clients[4], which is the specific benefit from DAS architecture. The testing area is a typical office room with various appliances (desks, chairs and computers). The RAUs were mounted on trolleys with height extensions, so the signals were generally radiated above all furniture. The measurements were carried out using the commercial software Networx, and was implemented by transferring large files between the Host PC and mobile laptops. In order to evaluate the throughput performance with fiber-length difference effects in the RoF DAS, we consider both the MIMO transmission and spatial diversity transmission. Seven configurations were used in our experiment, as shown in Table I using different groups of fiber links.

TABLE I. CONFIGURATIONS IN EXPERIMENT

\begin{tabular}{|c|c|c|c|}
\hline & AP Tyре & $F L^{1}-R A U 1(m)$ & $F L-R A U 2(m)$ \\
\hline Case1 & \multirow{5}{*}{ MIMO } & 25 & \multirow{4}{*}{25} \\
\hline Case2 & & 75 & \\
\hline Case 3 & & 125 & \\
\hline Case 4 & & 175 & \\
\hline Case 5 & & 125 & 125 \\
\hline Case6 & \multirow{2}{*}{ Spatial Diversity } & 25 & \multirow{2}{*}{25} \\
\hline Case 7 & & 175 & \\
\hline
\end{tabular}

\section{EXPERIMENTAL RESULTS}

In Cases 1-7, we measured the throughput in each location shown in the layout of Fig.2. At the CU, Belkin IEEE 802.11n MIMO-supported AP is used in Case 1-5, and 3com spatial diversity AP is used in Case 6-7. 


\section{A. Single client measurements}

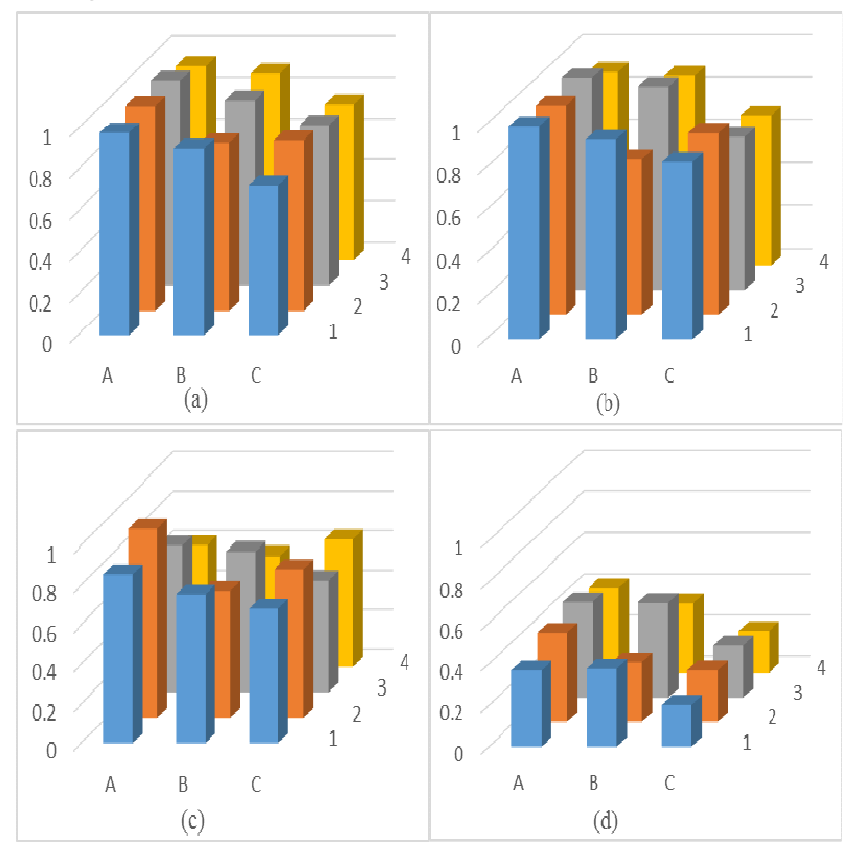

Fig. 3. MIMO-supported downlink throughput with different fiber links (a) Case 1, 25m-25m (b) Case 2,25m-75m (c) Case 3,25m-125m (d) Case 4, 25m$175 \mathrm{~m}$

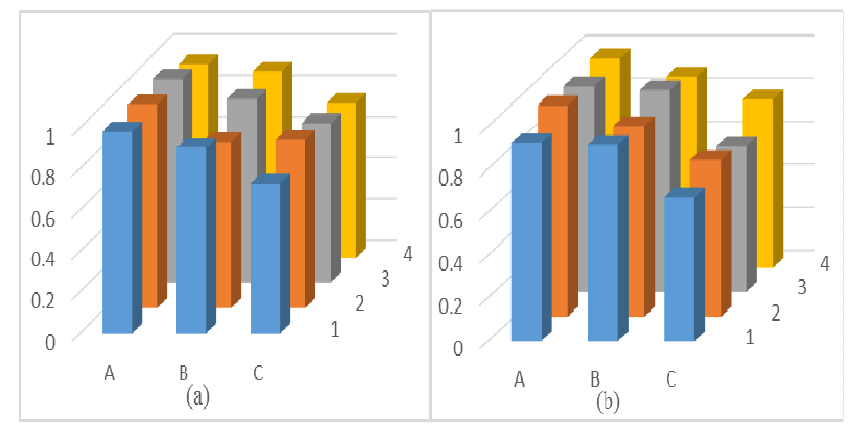

Fig. 4. MIMO-supported downlink throughput with the equal fiber links (a) Case $1,25 \mathrm{~m}-25 \mathrm{~m}$ (b)Case $5,125 \mathrm{~m}-125 \mathrm{~m}$

Fig.3 shows the normalized downlink throughput measurement results, which is normalized with the maximum throughput each mode can reach. (It should be noted that this is $96 \mathrm{Mbps}$ for the MIMO AP and $25 \mathrm{Mbps}$ for the spatial diversity AP, and that due to the use of MIMO techniques, the ratio is more that the $\times 2$ bandwidth ratio.) The measurements were implemented in each location inside the room with the different groups of fiber links. In Cases 2-4, the throughput dropped gradually as the fiber-length difference increased. This decrease is mostly because of the severe ISI, as the fixed fiber delay difference increases the interval between arrived signals from the two RAUs. As the channel degrades due to the large intervals between two spatial streams, the guard interval is insufficient to isolate the current symbol, when the fiber link difference is beyond a certain length. In Case 5, the throughput does not reduce much due to the extra equal fibre delay compared to Case 1, shown in Fig.4, while the heavier overhead causes a small performance decrease. Hence, this verifies that the throughput decrease was not caused by the fixed fiber delay, such as due to late arriving ACKs, but the nonalignment between two signal streams.

Fig.5 shows the result of throughput measurement with the spatial diversity AP, where only one antenna port is receiving at a time. We can observe that the throughput only shows very small changes between Case 6 and Case 7. This is simply because only the antenna which receives higher power or SNR is selected, and the poor channel state does not significantly affect the whole system performance.

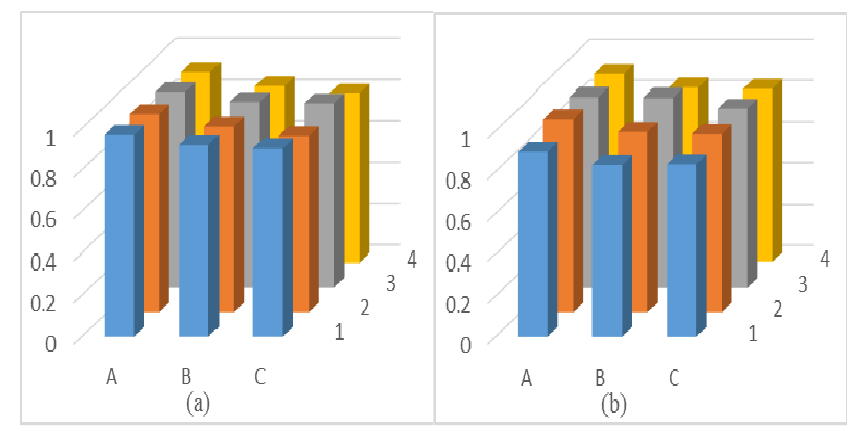

Fig. 5. Spatial Diversity-supported downlink throughput with different fibre links (a) $25 \mathrm{~m}-25 \mathrm{~m}$ (b) $25 \mathrm{~m}-175 \mathrm{~m}$

\section{B. Multiple clients measurements}

One additional client was added to determine the effects of multiple clients accessing the AP from both antenna ports. The same model laptops with same Network Interface Cards (NIC) were used to avoid any inequalities of throughput distribution, as different manufacturers often implement different MAC configurations which may cause extremely variable competition abilities. Additionally, the MIMO-supported AP was in $802.11 \mathrm{n}$ mode only, and no $802.11 \mathrm{~b} \& \mathrm{~g}$ clients were allowed to access the network. For the 802.11g diversity AP, no $802.11 \mathrm{~b}$ clients were present within the association radius of the AP, thus, we can ensure the maximum throughput was obtained.

Furthermore, we use the basic mode (two-handshake protocol) in the measurement and there was no hidden node problem. The experiments were carried out in different position groups. G1 represents both of the laptops are in the position $\mathrm{A} 1$ ( $\mathrm{A}$ is the row number and 1 is the column number), G2 is both in $\mathrm{A} 2, \mathrm{G} 3$ is both in B4, and G4 means 1 laptop in $\mathrm{A} 1$, the other one in $\mathrm{B} 4$. The data recordings were collected at the same time after the throughput distribution of the two laptops arrived at an equilibrium level.

In Fig.5 and Fig.6, MIMO-1 and Diversity-1 means the 1client downlink throughput measurement in the specific position or the average of two positions. Similarly, MIMO-2 and Diversity-2 represent the 2-client situations. A $10.7 \%$ $22.3 \%$ decrease of the aggregate throughput with the MIMO $\mathrm{AP}$ in $25 \mathrm{~m}-25 \mathrm{~m}$ situation when using 2 laptops can be observed in Fig.6, while 24.2\%-53.8\% decrease is observed in $25 \mathrm{~m}-175 \mathrm{~m}$ configuration in Fig.7. Greater throughput decrease was mainly due to more severe ISI and the blind 
collisions due to ineffective CSMA, when more clients access the AP through different RAUs. We can easily imagine that the aggregate throughput will be worse when more clients join the network as more collisions will occur. However, a slight increase with the diversity AP is apparent in Fig.6 and Fig.7 with more clients, as the utilization of the channel is higher. As a result of the reduced effect of additional fiber-length difference on this system, the diversity AP has better performance compared to the MIMO one in such cases. For the uplink, the performance followed the same general trends as the downlink.

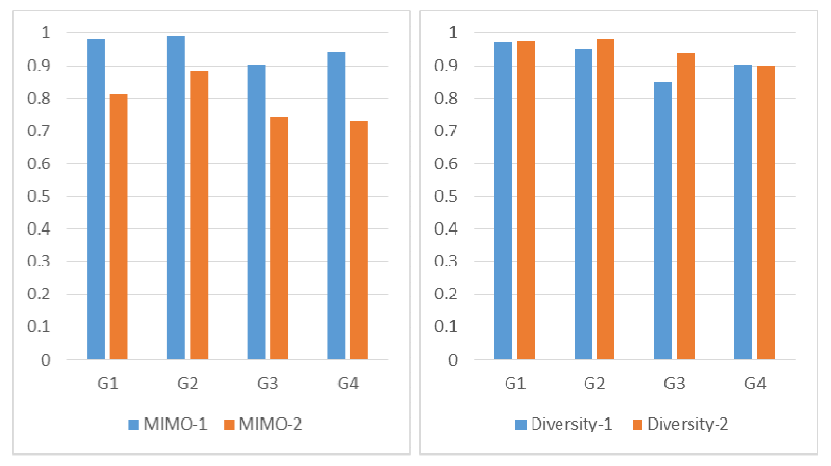

Fig. 6. Multiple users downlink throughput comparison for MIMO AP and Diversity AP respectively with the same fibre links $(25 \mathrm{~m}-25 \mathrm{~m})$

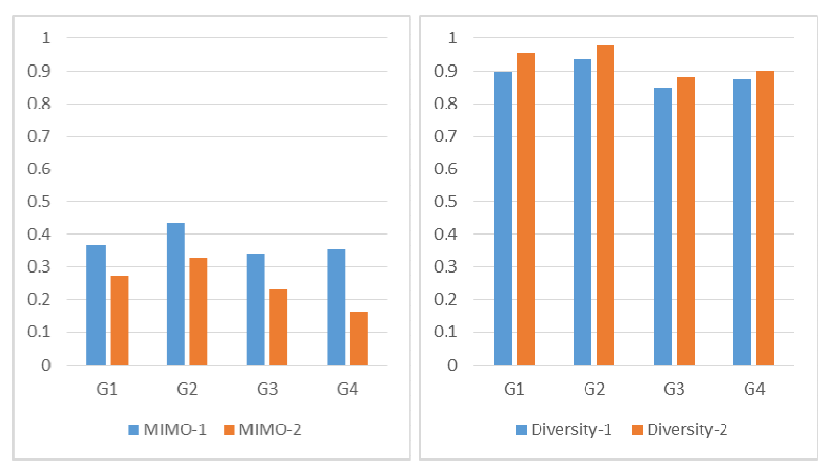

Fig. 7. Multiple users downlink throughput comparison for MIMO AP and Diversity AP respectively with different fibre links (25m-175m)

\section{CONCLUSION}

In this paper, we compared the throughput performance of MIMO signals and spatial diversity signals in a RoF-based
DAS with different fibre length links. The results show that MIMO signals can maintain high throughput performance when the fiber length difference between the two RAUs was under $100 \mathrm{~m}$ and dropped quickly when the fiber length difference was greater than this. For the spatial diversity signals, the high throughputs could be maintained even when the difference was $150 \mathrm{~m}$.

\section{ACKNOWLEDGMENT}

The authors are grateful to Anthony Nkansah for advice on the measurements, and to the China Scholarship Council for support for Y Fan.

\section{REFERENCES}

[1] T. Alade, H. Zhu, and H. Osman, "The impact of antenna selection and location on the performance of DAS in a multi-storey building," IEEE Wireless Communications and Networking Conference (WCNC), pp. $3213-3218,2013$

[2] H. Zhu, "Performance Comparison Between Distributed Antenna and Microcellular Systems," IEEE Journal on Selected Areas in Communications, pp. 1151 - 1163, 2011.

[3] P. Monteiro, S. Pato, E. López, D. Wake, N. J. Gomes, and A. Gameiro, "Fiber Optic Networks for Distributed Radio Architectures: FUTON Concept and Operation," IEEEWireless Communications and Networking Conference Workshops (WCNCW), pp. 1 - 5, 2010.

[4] K. Zhu, M. J. Crisp, S. He, and R. V. Penty, "MIMO system capacity improvements using radio-over-fibre distributed antenna system technology," Optical Fiber Communication Conference and Exposition and the National Fiber Optic Engineers Conference (OFC/NFOEC), pp. 1 - 3, 2011.

[5] T.Yamakami, T.Higashino, K.Tsukamoto, S.Komaki , "An Experimental Investigation of Applying MIMO to RoF Ubiquitous Antenna System," 2008 International Topical Meeting on Microwave photonics(MWP), pp.201 - 204,2008.

[6] P. Assimakopoulos, A. Nkansah, and N. J. Gomes, "Use of commercial Access Point employing spatial diversity in a Distributed Antenna Network with different fiber lengths," international topical meeting on Microwave photonics/ asia-pacific microwave photonics conference mwp/apmp, pp. 189 - 192, 2008.

[7] A. Das, M. Mjeku, A. Nkansah, and N. J. Gomes, "Effects on IEEE 802.11 MAC Throughput in Wireless LAN Over Fiber Systems," Journal of Lightwave Technology, pp. 3321 - 3328, 2007.

[8] G. S. D. Gordon, M. J. Crisp, R. V. Penty, and I. H. White, "Experimental investigation of antenna selection and transmit beamforming for capacity enhancement in $3 \times 3$ MIMO-enabled radioover-fiber DAS," International Topical Meeting on Microwave Photonics (MWP), pp. 313 - 316, 2013.

[9] "802.11n: The Next Generation of Wireless Performance", Cisco Public document. 